DESY $05-123$

FERMILAB-Pub-05/319-T

IFIC/05-26

ZU-TH 09-05

\title{
Determining Sneutrino Masses and Physical Implications
}

\author{
A. Freitas ${ }^{1}$, W. Porod ${ }^{2,3}$ And P. M. Zerwas ${ }^{4}$ \\ ${ }^{1}$ Fermi National Accelerator Laboratory, Batavia, IL 60510-500, USA \\ ${ }^{2}$ Universitat de Valéncia, 46071 Valéncia, Spain \\ ${ }^{3}$ Universität Zürich, CH-8057 Zürich, Switzerland \\ ${ }^{4}$ Deutsches Elektronen-Synchrotron DESY, D-22603 Hamburg, Germany
}

\begin{abstract}
In some areas of supersymmetry parameter space, sneutrinos are lighter than the charginos and the next-to-lightest neutralino, and they decay into the invisible neutrino plus lightest-neutralino channel with probability one. In such a scenario they can be searched for in decays of charginos that are pair-produced in $e^{+} e^{-}$collisions, and in associated sneutrino-chargino production in photon-electron collisions. The sneutrino properties can be determined with high accuracy from the edges of the decay energy spectra in the first case and from threshold scans in the second. In the final part of the report we investigate the mass difference of sneutrinos and charged sleptons between the third and the first two generations in seesaw-type models of the neutrino/sneutrino sector. For a wide range these mass differences are sensitive to the seesaw scale.
\end{abstract}




\section{Introduction}

The complex structure observed in the neutrino sector will have interesting consequences for the properties of the sneutrinos, the scalar supersymmetric partners of the neutrinos. These novel elements require the extension of the minimal supersymmetric Standard Model MSSM, e.g. by a superfield including the right-handed neutrino field and its scalar partner [1]. In particular, if the small neutrino masses are generated by the seesaw mechanism [2], a similar type of spectrum is induced in the scalar sector, splitting into light $\mathrm{TeV}$-scale and very heavy masses. Moreover, the intermediate seesaw scales will affect the evolution of the soft mass terms which break the supersymmetry at the high [GUT] scale, particularly in the third generation with large Yukawa couplings, so that universality will be broken at the low [electroweak] scale. This in turn will provide the opportunity to measure the intermediate seesaw scale of the third generation indirectly under well defined assumptions. Two sets of observables are of central interest:

- The masses of the charged and neutral sleptons within the first two generations are determined by the soft scalar mass parameters and the gaugino mass parameters at the unification scale, and the D-terms generated by the breaking of the grand unification and electroweak gauge symmetries.

- The mass differences between the sleptons of the third and the first two generations which are affected by the Yukawa couplings in the tau sector and the seesaw scale.

To develop a comprehensive picture, both the charged and the neutral sleptons must be analyzed with high precision in parallel.

Sneutrinos can be pair-produced in $e^{+} e^{-}$collisions. If they are heavier than the light chargino and the next-to-lightest neutralino, they can be searched for in the decays $\tilde{\nu}_{l} \rightarrow \nu_{l} \tilde{\chi}_{2}^{0}$ and $\tilde{\nu}_{l} \rightarrow l^{-} \tilde{\chi}_{1}^{+}$, since these final states generate visible signals in the detector. These channels have recently been studied in Refs. $[3,4]$ in detail. In contrast, if sneutrinos are lighter than these particles, they decay only to final states $\tilde{\nu}_{l} \rightarrow \nu_{l} \tilde{\chi}_{1}^{0}$ that are invisible and pair-production is useless for studying these particles. [Photon tagging of these pairs remains very difficult due to the reduced cross sections.]

However, in this configuration two other methods provide opportunities to study sneutrino masses:

- Chargino decays to sneutrinos and leptons,

$$
\tilde{\chi}_{1}^{ \pm} \rightarrow l^{ \pm} \tilde{\nu}_{l}^{(*)},
$$

with the charginos pair-produced in $e^{+} e^{-}$annihilation. These two-particle decays develop sharp edges at the endpoints of the lepton energy spectra. Sneutrinos of all the three generations can be explored this way.

- The first-generation sneutrino can also be studied in $e \gamma$ scattering [5]:

$$
e^{-} \gamma \rightarrow \tilde{\nu}_{e} \tilde{\chi}_{1}^{-} .
$$


The spectrum of Compton-backscattered laser light has a sharp peak at the maximum energy of the produced high-energy photons. In addition, the cross section for polarized $e, \gamma$ beams rises steeply at threshold, so that the scanning of the threshold region can be used to determine the sum of the $\tilde{\nu}_{e}$ and $\tilde{\chi}_{1}^{ \pm}$masses. Since, on the other hand, the mass of $\tilde{\chi}_{1}^{ \pm}$can be measured in other channels with high precision, the process (2) can serve as a channel for the $\tilde{\nu}_{e}$ mass measurement. However, standard $W$ production gives rise to a serious background problem and the photon spectrum must be controlled very carefully.

The slight shift of the parameters in the Snowmass point from SPS1a to SPS1a $[6,7]^{1}$ leads to a configuration in which the sneutrinos are lighter than the charginos and the secondlightest neutralino. We therefore have adopted the reference point SPS1a' for carrying out detailed analyses of sneutrino masses at a future linear collider [8].

The material presented in this report is divided into three sections. In the next section we develop the techniques for measuring the sneutrino masses in chargino decays, and in the subsequent section for scanning the threshold region of the inelastic SUSY Compton process. Detailed estimates of the expected errors on the sneutrino masses are presented in the reference point SPS1a' for both methods. In the final section some interesting physics implications are worked out. First, the D-terms are estimated from the mass difference between the charged and neutral sleptons. Going beyond the MSSM in the next step, we discuss in particular the variation of the mass differences between sleptons of the third and the first two generations with the Yukawa couplings and the intermediate seesaw scale. This analysis is carried out for a minimal $\mathrm{SO}(10)$ grand unification scenario with universal boundary conditions for the soft scalar mass parameters, but potentially modified by Dterms associated with the GUT gauge symmetry breaking. In this configuration the system of charged and neutral slepton masses determines the seesaw scale in addition to the complete set of universal soft mass parameters and D-terms at the GUT scale.

\section{Chargino Decays to Sneutrinos}

The relevant masses, widths and branching ratios for the reference point SPS1a' are listed in Tab. 1. In this reference point the branching ratio for the 2-body decay $\tilde{\chi}_{1}^{ \pm} \rightarrow \tilde{\nu}_{l}^{(*)} l^{ \pm}$ $(l=e, \mu)$ of the lightest chargino amounts to $13.4 \%$. With a production cross section of 100-200 fb not far above the threshold, a large number of sneutrinos can be generated. For a given $e^{+} e^{-}$energy the lepton decay energies are almost uniformly distributed between the minimum and maximum value $E_{\min }$ and $E_{\max }$ :

$$
E_{\min , \max }=\frac{\sqrt{s}}{4} \frac{m_{\tilde{\chi}_{1}^{ \pm}}^{2}-m_{\tilde{\nu}_{l}}^{2}}{m_{\tilde{\chi}_{1}^{ \pm}}^{2}}\left(1 \pm \sqrt{1-4 m_{\tilde{\chi}_{1}^{ \pm}}^{2} / s}\right)
$$

\footnotetext{
${ }^{1}$ The shift lowers the prediction for the cold dark matter density to the WMAP band.
} 


\begin{tabular}{|c||c|c|cc|}
\hline Sparticle & Mass $m[\mathrm{GeV}]$ & Width $\Gamma[\mathrm{GeV}]$ & \multicolumn{2}{|c|}{ Decay modes } \\
\hline \hline$\tilde{\nu}_{l}=\tilde{\nu}_{e} / \tilde{\nu}_{\mu}$ & 169.6 & 0.09 & $\tilde{\nu}_{l} \rightarrow \nu_{l} \tilde{\chi}_{1}^{0}$ & $100 \%$ \\
\hline$\tilde{\nu}_{\tau}$ & 167.8 & 0.15 & $\tilde{\nu}_{\tau} \rightarrow \nu_{\tau} \tilde{\chi}_{1}^{0}$ & $100 \%$ \\
\hline$\tilde{\tau}_{1}$ & 105.7 & 0.0037 & $\tilde{\tau}_{1} \rightarrow \tau \tilde{\chi}_{1}^{0}$ & $100 \%$ \\
\hline \hline$\tilde{\chi}_{1}^{0}$ & 100.8 & - & - & \\
\hline$\tilde{\chi}_{1}^{ \pm}$ & 180.5 & 0.074 & $\tilde{\chi}_{1}^{+} \rightarrow \tilde{\tau}_{1}^{+} \nu_{\tau}$ & $53 \%$ \\
& & & $\rightarrow \tilde{\nu}_{e} e^{+}$ & $13 \%$ \\
& & & $\rightarrow \tilde{\nu}_{\mu} \mu^{+}$ & $13 \%$ \\
& & & $\rightarrow \tilde{\nu}_{\tau} \tau^{+}$ & $19 \%$ \\
\hline
\end{tabular}

Table 1: Tree-level masses, widths and main branching ratios of sleptons and of the light neutralino and chargino states at Born level for the reference point SPS1a' [7].

These values determine the masses of the parent chargino and the child sneutrino:

$$
\begin{aligned}
m_{\tilde{\chi}_{1}^{ \pm}} & =\sqrt{s} \frac{\sqrt{E_{\min } E_{\max }}}{E_{\min }+E_{\max }}, \\
m_{\tilde{\nu}_{l}} & =m_{\tilde{\chi}_{1}^{ \pm}} \sqrt{1-\frac{2\left(E_{\min }+E_{\max }\right)}{\sqrt{s}}} .
\end{aligned}
$$

At the threshold for the production of the chargino pair the energy distribution is reduced to a sharp line.

The uniform distribution is distorted only slightly by polarization effects. Close to threshold the produced chargino $\tilde{\chi}_{1}^{ \pm}$is longitudinally and transversely polarized in the production plane with degrees [9]

$$
P_{\mathrm{L}}=-P_{0} \cos \theta \text { and } P_{\perp}=+P_{0} \sin \theta
$$

respectively, where $\theta$ denotes the polar production angle. The polarization degree $P_{0}$,

$$
P_{0}=\frac{Q_{\mathrm{L}}^{2}-Q_{\mathrm{R}}^{2}}{Q_{\mathrm{L}}^{2}+Q_{\mathrm{R}}^{2}},
$$

can be expressed by the bilinear $\mathrm{L} / \mathrm{R}$ charges

$$
\begin{aligned}
& Q_{\mathrm{L}}=2+\frac{D_{\mathrm{Z}}}{s_{\mathrm{W}}^{2} c_{\mathrm{W}}^{2}}\left(s_{\mathrm{W}}^{2}-\frac{1}{2}\right)\left(2 s_{\mathrm{W}}^{2}-\left|U_{11}\right|^{2}-\frac{1}{2}\left|U_{12}\right|^{2}-\left|V_{11}\right|^{2}-\frac{1}{2}\left|V_{12}\right|^{2}\right)-\frac{D_{\tilde{\nu}}}{4 s_{\mathrm{W}}^{2}}, \\
& Q_{\mathrm{R}}=2+\frac{D_{\mathrm{Z}}}{c_{\mathrm{W}}^{2}}\left(2 s_{\mathrm{W}}^{2}-\left|U_{11}\right|^{2}-\frac{1}{2}\left|U_{12}\right|^{2}-\left|V_{11}\right|^{2}-\frac{1}{2}\left|V_{12}\right|^{2}\right) .
\end{aligned}
$$

Here, $D_{\mathrm{Z}}=s /\left[s-M_{\mathrm{Z}}^{2}+i M_{\mathrm{Z}} \Gamma_{\mathrm{Z}}\right]$ and $D_{\tilde{\nu}}=s /\left[t-m_{\tilde{\nu}}^{2}\right]$ are the $Z$-boson s-channel and the $\tilde{\nu}_{e}$ t-channel exchange propagators, respectively, renormalized by the energy squared $s . U_{i j}$ and $V_{i j}$ are the mixing matrices of the negatively/positively charged charginos; for details see Ref. [10]. 


\begin{tabular}{|c|c|c|}
\hline Condition & Variable & Accepted range \\
\hline $\begin{array}{l}\text { Reject leptons in forward/ } \\
\text { backward region }\end{array}$ & lepton polar angle $\theta_{1}$ & $\begin{array}{l}\left|\cos \theta_{\mathrm{e}}\right|<0.90 \\
\left|\cos \theta_{\mu}\right|<0.95\end{array}$ \\
\hline $\begin{array}{l}\text { Reject soft leptons from radiative } \\
\text { photon splitting and } \gamma-\gamma \text { events }\end{array}$ & lepton energy $E_{1}$ & $E_{1}>5 \mathrm{GeV}$ \\
\hline $\begin{array}{l}\text { Reject missing momentum in for- } \\
\text { ward/backward region from parti- } \\
\text { cles lost in the beam pipe }\end{array}$ & $\begin{array}{l}\text { missing momentum polar } \\
\text { angle } \theta_{\vec{p}_{\text {miss }}}\end{array}$ & $\left|\cos \theta_{\vec{p}_{\text {miss }}}\right|<0.90$ \\
\hline $\begin{array}{l}\text { Angular separation between } \\
e \text { and } \mu \text { lepton }\end{array}$ & $\begin{array}{l}\text { angle } \phi_{\mathrm{e} \mu} \text { between electron } \\
\text { and muon }\end{array}$ & $\left|1-\cos \phi_{\mathrm{e} \mu}\right|>0.015$ \\
\hline
\end{tabular}

Table 2: Cuts to reduce the main Standard Model backgrounds and to account for the detector geometry and resolution.

With respect to the polarization axis the angular distribution of the lepton in the decay (1) of a completely polarized chargino must follow the $\cos \theta^{*}$ law as a consequence of angular momentum conservation,

$$
\frac{4 \pi}{\Gamma} \frac{\mathrm{d} \Gamma}{\mathrm{d} \cos \theta^{*}}=1-\cos \theta^{*},
$$

where $\theta^{*}$ is the lepton polar angle with respect to the chargino polarization axis in the chargino rest frame. Right-handedly polarized charginos decay to left-handedly polarized leptons for spin-flipping scalar couplings so that backward emission is dominant.

Near the threshold the degree $P_{0}$ of the chargino is close to unity. However, the longitudinal polarization which affects the lepton energy distribution, averages approximately to zero if integrated over the chargino production angle. Farther away from the threshold small polarization effects build up slowly. They can be calculated quantitatively by adopting the general analysis from Refs. [11, 9].

(A) Assuming the two first and second generation L-sneutrinos, $\tilde{\nu}_{e}$ and $\tilde{\nu}_{\mu}$, to be massdegenerate, a final state $e \mu+\not \subset$, including one electron, one muon and missing energy carried away by the neutrinos and neutralinos, is little contaminated by backgrounds. The primary background source from Standard Model processes are $W^{+} W^{-}$pair production and single $W$ production, with the $W$ 's decaying into a charged lepton and the associated neutrino, $W^{\mp} \rightarrow l_{\stackrel{(-)}{\nu})_{l}}$.

Due to spin correlations and the boost factor, the background from $W$ bosons tends to peak along the beam direction and can be reduced by requiring both charged leptons in the central detector region. A simple set of cuts, summarized in Tab. 2, also reduces backgrounds from soft and collinear photon contributions and tau-induced backgrounds below the per-cent level.

Besides direct chargino and $W$-boson decays into electrons and muons, the $e \mu+\not E$ signature can also be generated by leptonic tau decays. In the numerical analysis, cascade decays 
via intermediate tau leptons have been included both for the chargino production process and the $W$ background.

Several other supersymmetric channels are open which give rise to $e \mu+\not E$ final states through tau decays. The cross section for $\tilde{\tau}$ pair production, with $\tilde{\tau}$ decaying to $\tau \tilde{\chi}_{1}^{0}$, is of similar size as the signal cross section after the leptonic tau branchings are included; however, cascading down to the $e$ and $\mu$ final leptons in two steps, the energies are softened to small values. Therefore we do not expect this channel to have a significant impact on the determination of neither the upper nor the lower edges of the signal lepton distributions. Nevertheless, this channel has been included explicitly in the numerical calculation. Cascades from chargino and neutralino pairs to $e, \mu$ leptons through taus are doubly suppressed by the leptonic tau branching ratios, cf. Ref.[12], and they involve at least two consecutive decay steps so that the energy distribution is doubly soft. These background channels could therefore be neglected in the present analysis.

Results for the lepton energy distributions are shown in Fig. 1 for the signals and the backgrounds. The energy has been chosen at $450 \mathrm{GeV}$, where the cross section is maximal, and an integrated luminosity of $500 \mathrm{fb}^{-1}$ has been assumed. The signal spectrum shows the expected almost uniformly flat distribution. The edges are slightly rounded due to initialstate radiation and beamstrahlung. Backgrounds from $W$-boson production are relatively small and flat so that the kinematical edges of the signal distribution are not distorted. The position of the edges $E_{\min }$ and $E_{\max }$ can be extracted from a simple fit using a box-shaped fit function convoluted with the initial-state radiation and beamstrahlung spectra. For the reference point SPS1a' the fit to the electron energy spectrum yields well determined values from which the chargino and sneutrino masses can be derived:

$$
\begin{aligned}
E_{\text {min }, \mathrm{e}} & =5.30_{-0.06}^{+0.07} \mathrm{GeV}, & E_{\max , \mathrm{e}} & =21.04_{-0.07}^{+0.07} \mathrm{GeV}, \\
m_{\tilde{\chi}_{1}^{ \pm}} & =180.4_{-0.7}^{+0.7} \mathrm{GeV}, & m_{\tilde{\nu}_{e}} & =169.5_{-0.6}^{+0.7} \mathrm{GeV} .
\end{aligned}
$$

Similarly the muon energy spectrum is fitted with the results

$$
\begin{aligned}
E_{\min , \mu} & =5.30_{-0.07}^{+0.08} \mathrm{GeV}, & E_{\max , \mu} & =21.06_{-0.07}^{+0.07} \mathrm{GeV} \\
m_{\tilde{\chi}_{1}^{ \pm}} & =180.4_{-0.7}^{+0.8} \mathrm{GeV}, & m_{\tilde{\nu}_{\mu}} & =169.5_{-0.7}^{+0.8} \mathrm{GeV}
\end{aligned}
$$

Note that the accuracy of the chargino mass corresponds to the value expected from a threshold scan [13], $\delta_{\text {thr }} m_{\tilde{\chi}_{1}^{ \pm}}=0.55 \mathrm{GeV}$. Combining the two methods of chargino mass measurement, the accuracies sharpen to

$$
\delta m_{\tilde{\chi}_{1}^{ \pm}} \approx \pm 0.45 \mathrm{GeV} \quad \text { and } \quad \delta m_{\tilde{\nu}_{e, \mu}} \approx \pm 0.40 \mathrm{GeV} .
$$

Thus a very high accuracy can be reached in this configuration.

(B) A special case are chargino decays to $\tilde{\nu}_{\tau}$ and $\tau$. Since the tau decays into an invisible neutrino and a charged pion, rho meson or other low-mass hadrons, the energy distribution of the visible meson is not uniform but follows asymptotically the familiar relation $\log \left(1 / x_{\text {had }}\right)$ for the (observed) fraction $x_{\text {had }}$ of the $\tilde{\chi}_{1}^{ \pm}$energy. This distribution does not generate a sharp 
(a)

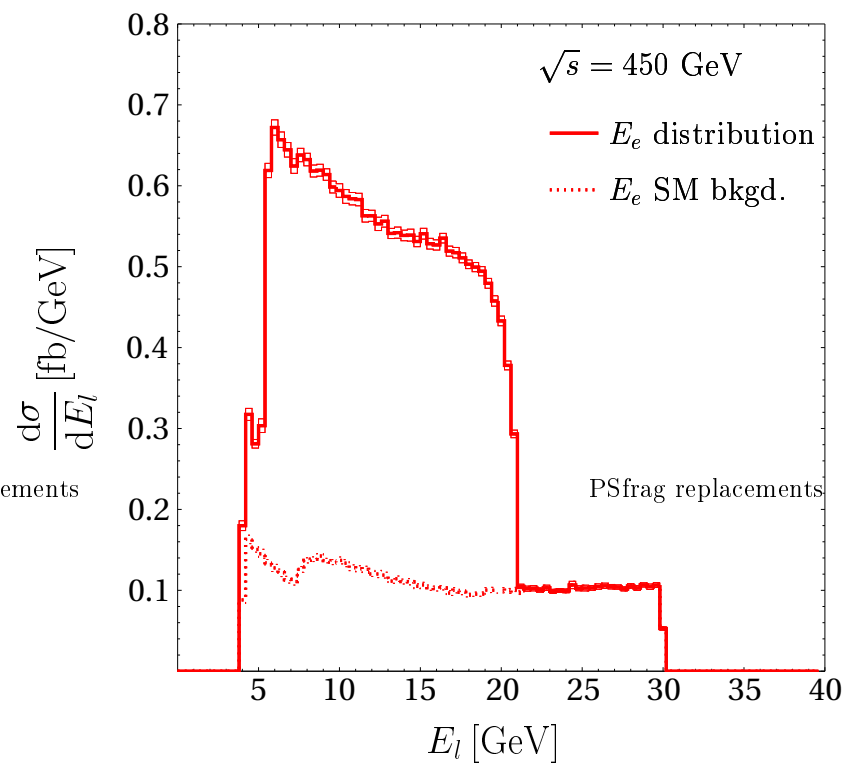

(b)

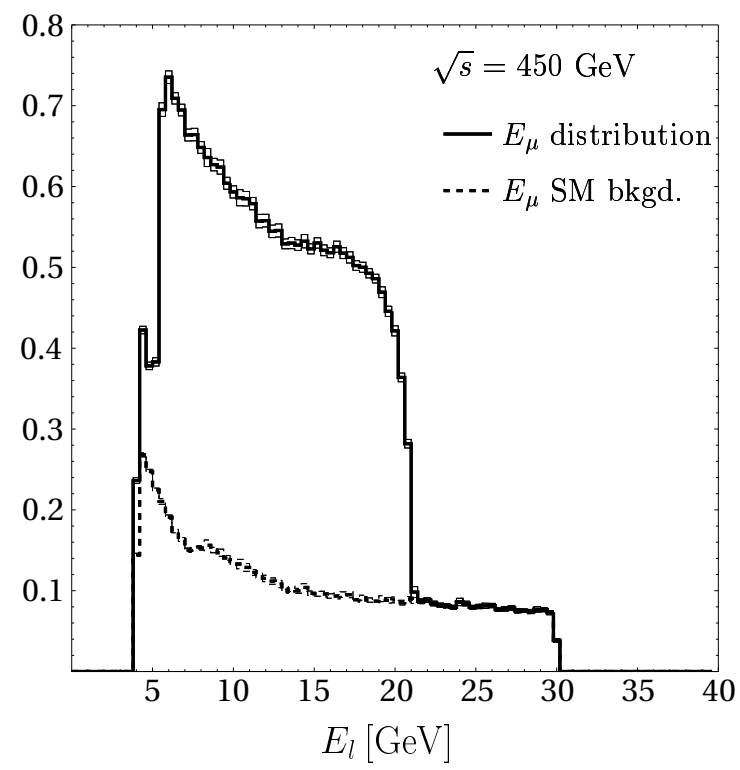

Figure 1: (a) Electron and (b) muon energy distributions for the process $e^{+} e^{-} \rightarrow\left(\tilde{\chi}_{1}^{+} \tilde{\chi}_{1}^{-}\right) \rightarrow$ $e^{ \pm} \mu^{\mp} \tilde{\nu}_{e} \tilde{\nu}_{\mu} \rightarrow e^{ \pm} \mu^{\mp}+\not$. The signals for the lepton energy spectra are shown over the background from Standard Model sources. Cuts of $4 \mathrm{GeV}$ and $30 \mathrm{GeV}$ are included for the lepton energies.

(a)

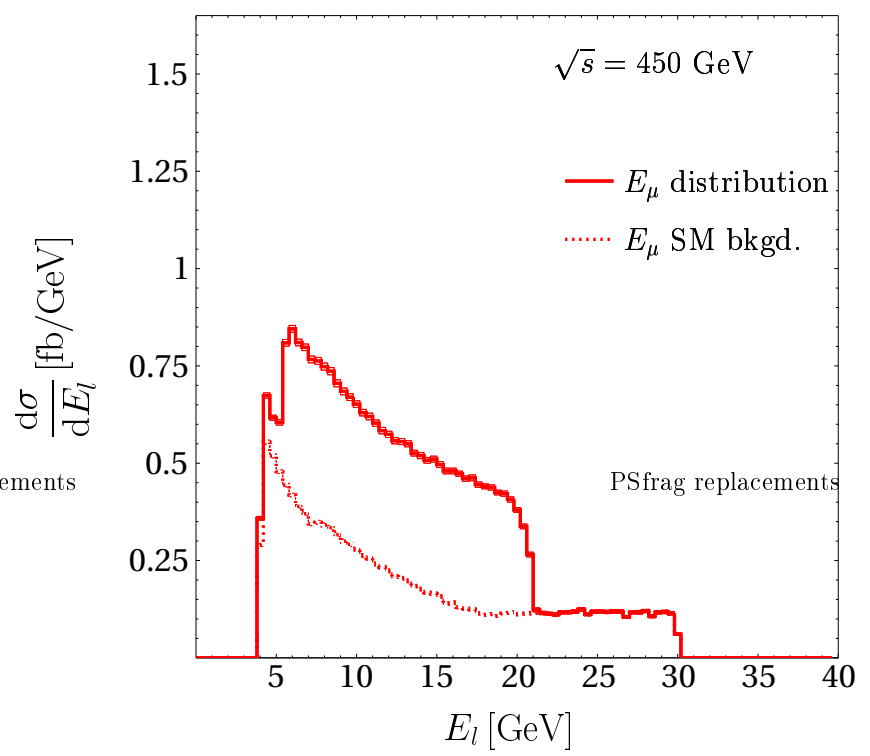

(b)

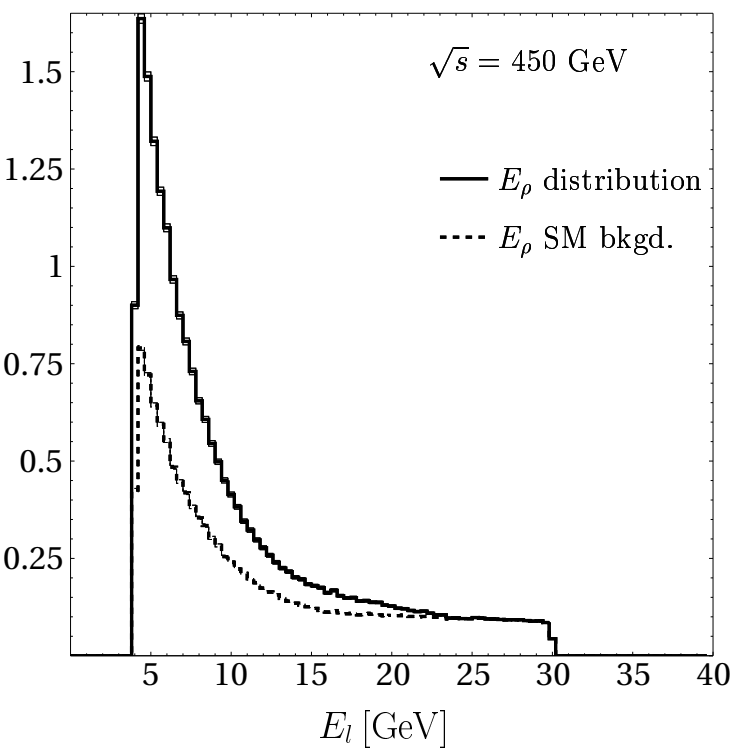

Figure 2: (a) Muon and (b) tau hadron energy distributions for the process $e^{+} e^{-} \rightarrow$ $\left(\tilde{\chi}_{1}^{+} \tilde{\chi}_{1}^{-}\right) \rightarrow \mu^{ \pm} \tau^{\mp} \tilde{\nu}_{\mu} \tilde{\nu}_{\tau} \rightarrow \mu^{ \pm} \tau^{\mp}+\not$. As in Fig. 1, Standard Model backgrounds are shown seperately and cuts of $4 \mathrm{GeV}$ and $30 \mathrm{GeV}$ are included for the lepton energies. 
edge anymore as in uniform energy distributions so that the error in $\tilde{\nu}_{\tau}$ mass measurements increases correspondingly.

In the following, all hadronic tau decay channels with a total branching ratio of $65 \%$ are included. For simplicity, the tau decay kinematics is approximated by the dominant decay into a rho meson. The upper tail of the decay energy spectrum for other important hadronic tau decay modes into one or three pions or into the $a_{0}$ meson are very similar to the rho meson spectrum, so that this approximation is sufficient for the purpose of this analysis. The same standard cuts listed in Tab. 2 for the muon are applied for the final state rho meson, only with a stricter cut on the polar angle, $\left|\cos \theta_{\rho}\right|<0.8, E_{\rho}>5 \mathrm{GeV},\left|1-\cos \phi_{\mathrm{l} \rho}\right|>0.015$. The upper endpoint of the rho meson energy spectrum is the same as the maximum energy $E_{\max , \tau}$ of the tau coming from the chargino decay, as given by eq. (3).

In addition to the decay of the charginos into $\tilde{\nu}_{\tau}$ and $\tau$, the decay into a charged stau and neutrino, $\tilde{\chi}_{1}^{ \pm} \rightarrow \tilde{\tau}_{1}^{ \pm} \stackrel{(-)}{\nu_{\tau}}$, is also possible in the SPS1a' scenario (see Tab. 1). However, the maximum energy of a tau lepton originating from the latter decay is smaller, about $15.8 \mathrm{GeV}$. This value is well below the upper endpoint of the tau energy spectrum from the sneutrino decay chain, $\tilde{\chi}_{1}^{ \pm} \rightarrow \tilde{\nu}_{\tau}^{(*)} \tau^{ \pm}, E_{\max , \tau}=24.5 \mathrm{GeV}$, so that this endpoint is not contaminated by the $\tilde{\tau}_{1}$ background ${ }^{2}$.

For analyzing the tau-sneutrino decay chain, it is expedient to consider the final state $\mu \tau+\not B$, which has lower background levels than the $e \tau+\not \mathcal{E}$ final state, since the single $W$ process does not contribute.

As before, only $\tilde{\tau}$ pair production is taken into account explicitly as a supersymmetric background channel. Neutralino pairs leading to $\tau \mu+\not E$ are suppressed and accumulate at small energies. However, as the tau signal channel does not generate sharp edges in the visible $\rho$ energy distribution anymore, we focus the analysis on the clean upper onset of the $\rho$ spectrum. Since all the parameters of the background $\tilde{\tau}$ channel can be pre-determined independently with high precision [14], this contribution can be calculated reliably.

The energy distributions for the muon and the hadronic tau decay products [assuming the decay $\tau \rightarrow \rho \nu_{\tau}$ ] are shown in Fig. 2 for signal and Standard Model and supersymmetric background channels.

A fit to the upper edge of muon energy spectrum yields $E_{\max , \mu}=21.0 \pm 0.1 \mathrm{GeV}$, in agreement with the previous estimate. The rho meson energy endpoint can be extracted by a fit to Monte-Carlo template samples. From the upper tail of the rho meson spectrum one obtains for the energy spectrum endpoint

$$
E_{\max , \tau}=24.5_{-1.0}^{+1.0} \mathrm{GeV} .
$$

Using the chargino mass extracted from the muon distribution, eq. (4), as an input, the $\tilde{\nu}_{\tau}$ mass can be derived from this endpoint,

$$
m_{\tilde{\nu}_{\tau}}=167.6_{-0.8}^{+0.9} \mathrm{GeV} \text {. }
$$

\footnotetext{
${ }^{2}$ This is generically expected if the $\tilde{\tau}_{1}-\tilde{\chi}_{1}^{0}$ mass difference is significantly smaller than the $\tilde{\nu}_{\tau}-\tilde{\chi}_{1}^{ \pm}$mass difference. In other scenarios, however, the chargino decays into staus might make the extraction of the tau-sneutrino signal virtually impossible.
} 
All errors quoted above are statistical errors only. Nevertheless, the results derived in this analysis are clearly encouraging. [A detailed experimental simulation including systematic errors is beyond the scope of our analysis.]

\section{$3 \quad e \gamma$ Production of Sneutrinos}

Electron sneutrinos can also be studied in associated production with charginos in electronphoton collisions [5]. The photon beam is generated through laser light scattering on the second incoming electron beam [15]. If circularly polarized laser photons are back-scattered off electrons of opposite-sign helicity, the spectrum of the generated high-energy photons has a sharp maximum at the kinematical edge,

$$
E_{\gamma, \max }=\frac{E_{\mathrm{b}}}{1+x^{-1}}
$$

with

$$
x=\frac{4 E_{0} E_{\mathrm{b}}}{m_{e}^{2}} \simeq 4.8,
$$

where $E_{0}$ denoting the laser photon energy, $E_{\mathrm{b}}$ the electron beam energy and $E_{\gamma}$ the resulting scattered photon energy; the ratio $E_{\gamma, \max } / E_{\mathrm{b}}$ is close to 0.8 for standard electron and laser energies. The high-energy photons are circularly polarized themselves at the edge with helicity opposite to the helicity of the laser photons.

If in the subsequent $e \gamma$ collision process electron and photon helicities are chosen of equal sign, the final-state system is generated in an S-wave near the threshold. The cross section therefore rises sharply at the threshold proportional to the velocity $\beta$ of the sneutrino [5],

$$
\sigma\left[e_{\mathrm{L}}^{-} \gamma_{\mathrm{L}} \rightarrow \tilde{\nu}_{e} \tilde{\chi}_{1}^{-}\right]=\frac{2 \pi \alpha m_{\tilde{\chi}_{1}^{ \pm}}^{2} \beta}{s^{2}} \frac{V_{11}^{2}}{s_{\mathrm{W}}^{2}} \times \sum_{\lambda= \pm 1}(1+\lambda \beta)\left[\sqrt{\left(1-\beta^{2}\right) s / m_{\tilde{\chi}_{1}^{ \pm}}^{2}}-(1+\lambda \beta)\right]^{2}+\mathcal{O}\left(\beta^{2}\right) .
$$

Thus traversing the energy threshold for the supersymmetric Compton process leads to the onset of a striking increase of the observed production rate. If the chargino mass has been pre-determined, the threshold energy,

$$
E_{\mathrm{thr}}=m_{\tilde{\chi}_{1}^{ \pm}}+m_{\tilde{\nu}_{e}}
$$

can be exploited to extract the electron-sneutrino mass.

This theoretical picture is modified in a more realistic analysis of the spectrum of the back-scattered laser photons. During the beam collisions the electrons are strongly deflected by the opposite electron beam. As a result, the er luminosity is reduced compared to the naive expectation without repulsion. Due to the deflection away from the axis, the electrons do not collide in general with the highest energy photons, but with those of somewhat lower 
energy. Furthermore, the simultaneous absorption of more than one laser photon generates additional peaks at high photon energies.

These effects can be calculated by Monte Carlo beam simulations [16]. We have adopted for our analysis the convenient parametrization of Ref. [17], which has been adjusted to detailed simulations and which accounts well for the characteristics of the spectrum. The maximum energy of the primary peak is reduced compared with the value in the single Compton process, and a second but small peak develops at high energies. The primary edge is by far dominant and it is very pronounced so that on the whole a sharp onset is still guaranteed despite of the complicated interactions between the electron and photon field. Moreover, the edge can be calibrated by scanning the threshold for $e^{-} \gamma \rightarrow \tilde{e}^{-} \tilde{\chi}_{1}^{0}$ production which can be predicted very accurately from $e^{+} e^{-}$data and which can therefore be exploited to control the beam spread parameters near the maximum of the distribution.

The signal for associated sneutrino-chargino production in $e \gamma$ collisions is characterized by a single isolated lepton coming from the chargino decay (1) plus missing energy. The muon final state,

$$
e^{-} \gamma \rightarrow \tilde{\nu}_{e} \tilde{\chi}_{1}^{-} \rightarrow \tilde{\nu}_{e} \tilde{\nu}_{\mu}^{*} \mu^{-}
$$

is less contaminated by backgrounds than the electron final state and leads to experimentally cleaner final states than tau which involves subsequent decay channels. Moreover, the supersymmetric background channel $\tilde{e} \tilde{\chi}_{1}^{0} \rightarrow e+\tilde{\chi}_{1}^{0} \tilde{\chi}_{1}^{0}$ is eliminated from the $\mu$ sample. Therefore in the following only the muon final state will be studied. Large backgrounds arise from single $W$-boson production,

$$
e^{-} \gamma \rightarrow \nu_{e} W^{-} \rightarrow \nu_{e} \bar{\nu}_{\mu} \mu^{-}
$$

For the sneutrino signal, the muon energy is relatively small due to the small mass difference $m_{\tilde{\chi}_{1}^{ \pm}}-m_{\tilde{\nu}_{\mu}}$, whereas the $W$ background leads to larger values for the muon energy $E_{\mu}$. This distinction is somewhat washed out by the photon energy spectrum, but a cut $E_{\mu}<25 \mathrm{GeV}$ is still effective to reduce the background drastically. In addition, the decay muon from the $W$ boson is peaked in the forward direction, $\cos \theta>0$, where $\theta$ is the angle between the direction of the incoming electron beam and the outgoing muon. Consequently, by applying a cut on the scattering angle, $\cos \theta<0$, the signal-to-background is further improved. As before, it is also required that the muon has a minimal energy of $5 \mathrm{GeV}$ and is emitted into the central region of the detector, $\left|\cos \theta_{\mu}\right|<0.95$.

In Fig. 3, the onset of the sneutrino-chargino threshold is shown after convolution with the photon energy spectrum of Ref. [17]; beamstrahlung and initial-state radiation for the $e^{-}$beam are included. Also shown is the remaining background after applying the cuts introduced above. As explained before, the signal is enhanced by choosing left-polarized $e^{-}$beams and right-handed polarization for the laser photons, resulting in a left-polarized photon beam near the kinematical edge. A polarization degree of $90 \%$ is assumed for the $e^{-}$ beams, while the laser source is taken to be $100 \%$ polarized.

As evident from the figure, the background is still significantly larger than the sneutrinochargino signal, but the expected rates are large, thus allowing a statistical distinction even 


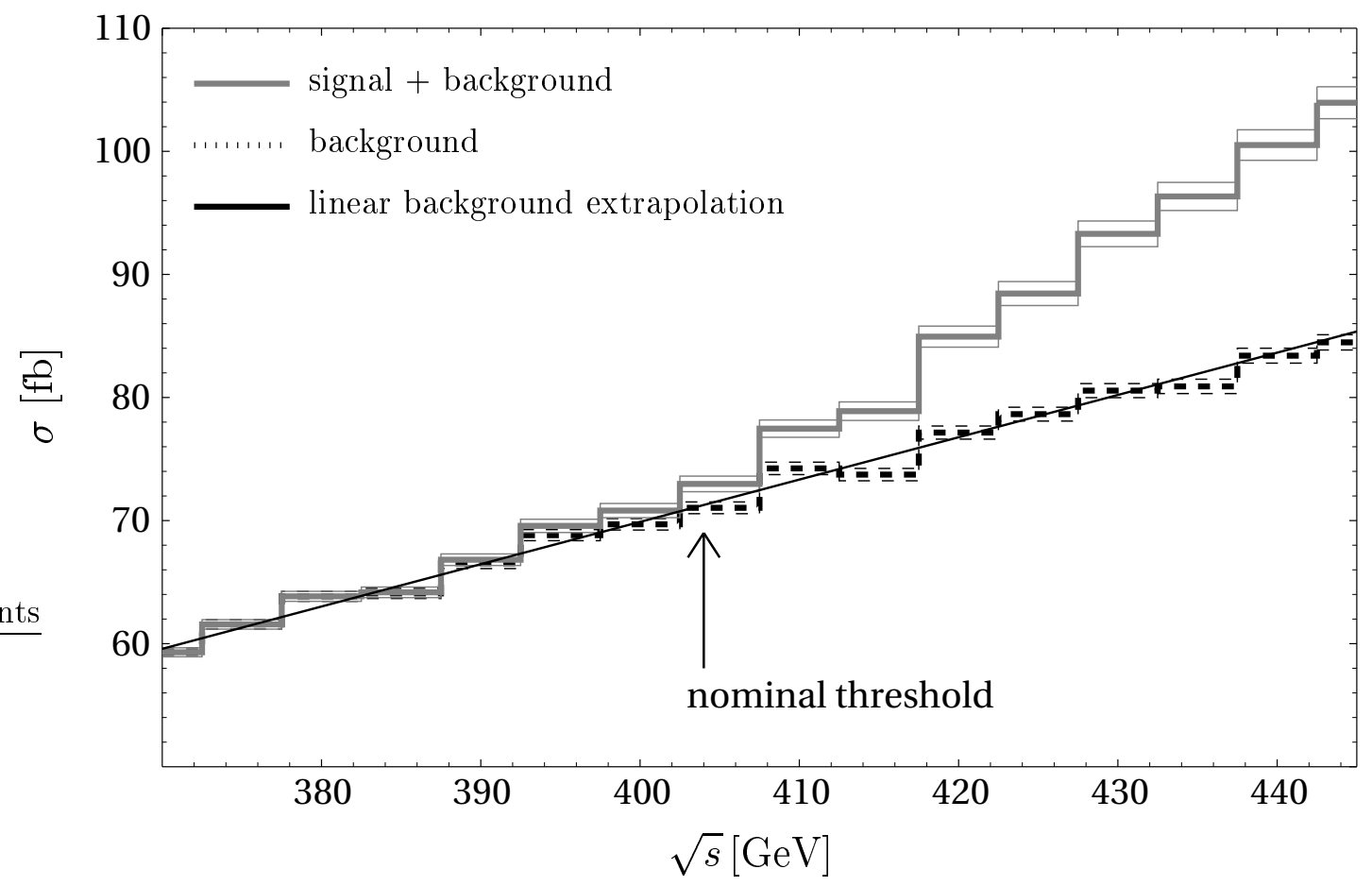

Figure 3: The excitation curve for $e^{-} \gamma \rightarrow \tilde{\chi}_{1}^{-} \tilde{\nu}_{e}$ production over the dominant Standard Model background as a function of the center-of-mass energy of the two incoming electron beams (one of which is used for generating the high-energy photons). The plot has been generated including the photon beam spectrum, beamstrahlung and initial-state-radiation for the electron beam, polarization of both beams and cuts to reduce the background.

in this situation. The dependence of the background on the $e^{-} e^{-}$center-of-mass energy $\sqrt{s}$ can be well described by a linear approximation, so that it can be extrapolated from measurements at values of $\sqrt{s}$ below the onset of the signal, as indicated in the figure.

We estimate the precision for the sneutrino mass measurement from a threshold scan based on simulated data at six equidistant scan points in the range $\sqrt{s}=380$ to $430 \mathrm{GeV}$ for the initial $e^{-} e^{-}$energy. An integrated luminosity of $10 \mathrm{fb}^{-1}$ is assumed to be spent on each scan point. The sneutrino mass is extracted using a binned likelihood method. Taking into account the chargino mass measurement error from a $\tilde{\chi}_{1}^{+} \tilde{\chi}_{1}^{-}$threshold scan, $\delta_{\text {thr }} m_{\tilde{\chi}_{1}^{ \pm}}=0.55$ $\mathrm{GeV}$, this leads to

$$
m_{\tilde{\nu}_{e}}=169.8 \pm 3.2 \mathrm{GeV} .
$$

While the precision achieved by this method is worse than the mass determination from chargino decay spectra elaborated in section 2, the $e \gamma$ threshold scan nevertheless provides an interesting alternative to search for invisibly decaying particles and to determine their mass with per-cent level accuracy. It may also be viewed as a complementary check of decay spectra measurements. 


\section{Implications: Determining GUT parameters and the seesaw scale}

Complementing the precision measurements of the charged slepton masses by the sneutrino masses of all three generations has exciting consequences for exploring physics scenarios at high scales. This includes potentially the universality of the soft scalar mass parameters, Dterms associated with the breaking of the grand unification gauge theory, and last-not-least the size of the seesaw scale in neutrino physics.

We will illustrate these points in an $\mathrm{SO}(10)$ model $^{3}$ in which the matter superfields of the three generations belong to 16-dimensional representations of $\mathrm{SO}(10)$ and the standard Higgs superfields to 10-dimensional representations while a Higgs superfield in the 126-dimensional representation generates Majorana masses to the right-handed neutrinos. As a result, the Yukawa couplings in the neutrino sector are proportional to the up-type quark mass matrix, for which the standard texture is assumed. The $\mathrm{SO}(10)$ symmetry is broken to the Standard Model $\mathrm{SU}(3) \times \mathrm{SU}(2) \times \mathrm{U}(1)$ symmetry at the grand unification scale $M_{U}$ directly. We will assume universality for the sfermion mass parameters at $M_{U}$, yet potentially modified by D-terms of the order of the electroweak scale [18]:

$$
\begin{aligned}
& m_{\mathrm{E}}^{2}=m_{16}^{2}+D_{U}, \\
& m_{\mathrm{L}}^{2}=m_{16}^{2}-3 D_{U}, \\
& m_{\mathrm{R}}^{2}=m_{16}^{2}+5 D_{U} .
\end{aligned}
$$

$m_{\mathrm{E}}, m_{\mathrm{L}}$ and $m_{\mathrm{R}}$ denote the iso-singlet, -doublet and R-sneutrino scalar mass parameters, $m_{16}$ the universal scalar mass, and $D_{U}$ the $\mathrm{SO}(10)$ D-term contribution. For simplicity we identify the soft breaking masses of the Higgs sector also with $m_{16}$; this technical simplification can easily be relaxed.

Assuming that the Yukawa couplings are the same for up-type quarks and neutrinos at the GUT scale and that the Majorana mass matrix of the right-handed neutrinos has a similar structure, one obtains a (weakly) hierarchical neutrino mass spectrum and nearly bi-maximal mixing for the left-handed neutrinos [19]. ${ }^{4}$ In this class of models the masses of the right-handed neutrinos are also hierarchical ${ }^{5}$,

$$
M_{\mathrm{R}_{3}}: M_{\mathrm{R}_{2}}: M_{\mathrm{R}_{1}} \sim m_{t}^{2}:\left(\kappa_{c} m_{c}\right)^{2}:\left(\kappa_{u} m_{u}\right)^{2}
$$

with $\kappa$ 's of order 10 . The overall scale is set by $m_{t}$ and the mass of the heaviest neutrino:

$$
M_{\mathrm{R}_{3}} \sim m_{t}^{2} / m_{\nu_{3}}
$$

\footnotetext{
${ }^{3}$ The pattern of mass ratios among the supersymmetric particles remains analogous to the SPS1a' model so that measurement errors can be obtained by scaling from the preceding sections.

${ }^{4}$ The neutrino Yukawa couplings induce off-diagonal elements in $M_{L}^{2}$ and $A_{l}$ leading to flavour changing sneutrino decays. However, as we will see later, these are CKM suppressed and can, thus, safely be neglected in the following.

${ }^{5}$ These relations are supposed to hold only at the level of very rough estimates; the only crucial point is the outstanding size of the $\mathrm{R}_{3}$ mass.
} 
For $m_{\nu_{3}} \sim 5 \times 10^{-2} \mathrm{eV}$, the heavy neutrino mass of the third generation amounts to $\sim 6 \times 10^{14}$ $\mathrm{GeV}$, i.e. a value close to the grand unification scale $M_{U}$.

Even though the soft mass parameters are assumed to be of the order of the electroweak scale or slightly above, the seesaw mechanism is induced also in the scalar sneutrino sector through the Higgs-126 couplings in the superpotential, see e.g. [20, 21],

$$
m_{\tilde{\nu}_{k \mathrm{R}}}^{2} \simeq m_{\mathrm{R}_{k}}^{2}
$$

so that R-sneutrinos cannot be generated at colliders.

The $\mathrm{SO}(10)$ gauge invariance requires a unified gaugino mass parameter $M_{1 / 2}$ at the scale $M_{U}$. We choose the high-scale supersymmetry breaking parameters in the framework of the SPS1a' scenario,

$$
M_{1 / 2}=250 \mathrm{GeV}, \quad m_{16}=70 \mathrm{GeV}, \quad A_{0}=-300 \mathrm{GeV}, \quad D_{U}=0
$$

and

$$
\tan \beta=10, \quad \mu>0 .
$$

This minimalistic model is compatible with all the observations in the neutrino sector. It may serve to illustrate the potential of precision measurements of charged and neutral scalar lepton masses for exploring physics close to grand unification/Planck scale.

To leading order of the solutions of the renormalization group equations ${ }^{6}$ the masses of the scalar selectrons and $e$-sneutrino can be expressed by the high scale parameters $m_{16}$ and $M_{1 / 2}$, and the D-terms:

$$
\begin{aligned}
m_{\tilde{e}_{R}}^{2} & =m_{16}^{2}+D_{U}+\alpha_{R} M_{1 / 2}^{2}-s_{W}^{2} M_{Z}^{2} \cos 2 \beta, \\
m_{\tilde{e}_{L}}^{2} & =m_{16}^{2}-3 D_{U}+\alpha_{L} M_{1 / 2}^{2}-\left(1 / 2-s_{W}^{2}\right) M_{Z}^{2} \cos 2 \beta, \\
m_{\tilde{\nu}_{e L}}^{2} & =m_{16}^{2}-3 D_{U}+\alpha_{L} M_{1 / 2}^{2}+1 / 2 M_{Z}^{2} \cos 2 \beta .
\end{aligned}
$$

The coefficients $\alpha_{R}$ and $\alpha_{L}$ can be determined numerically and the integration of the 1-loop RGEs yields $\alpha_{R} \simeq 0.15$ and $\alpha_{L} \simeq 0.5$. Analogous representations can be derived, to leading order, for the scalar masses of the third generation, complemented however by additional contributions $\Delta_{\tau}$ and $\Delta_{\nu_{\tau}}\left[M_{\mathrm{R}}\right]$ from the standard tau Yukawa term and the Yukawa term in the tau neutrino sector, respectively:

$$
\begin{aligned}
m_{\tilde{\tau}_{R}}^{2} & =m_{16}^{2}+D_{U}+\alpha_{R} M_{1 / 2}^{2}-s_{W}^{2} M_{Z}^{2} \cos 2 \beta-2 \Delta_{\tau}+m_{\tau}^{2} \\
m_{\tilde{\tau}_{L}}^{2} & =m_{16}^{2}-3 D_{U}+\alpha_{L} M_{1 / 2}^{2}-\left(1 / 2-s_{W}^{2}\right) M_{Z}^{2} \cos 2 \beta-\Delta_{\tau}-\Delta_{\nu_{\tau}}+m_{\tau}^{2}, \\
m_{\tilde{\nu}_{\tau}}^{2} & =m_{16}^{2}-3 D_{U}+\alpha_{L} M_{1 / 2}^{2}+1 / 2 M_{Z}^{2} \cos 2 \beta-\Delta_{\tau}-\Delta_{\nu_{\tau}} .
\end{aligned}
$$

\footnotetext{
${ }^{6}$ One-loop corrections are properly accounted for in the numerical analyses; they cancel to a large extent if mass differences are calculated.
} 
[The factor 2 in front of $\Delta_{\tau}$ in Eq. (36) is due to the fact that the $S U(2)$ doublet is propagating in the loop.] The contribution $\Delta_{\nu_{\tau}}$ carries the information on the value of the heavy righthanded neutrino mass. As will be shown below, this parameter is proportional to the Yukawa coupling squared in the $\nu_{\tau}$ sector which, within the seesaw mechanism, is linear in the heavy neutrino mass of the third generation. Since both parameters $\Delta_{\tau}$ and $\Delta_{\nu_{\tau}}$ are positive, the masses in the stau sector are shifted downwards compared to the masses in the selectron sector. The shift is enhanced by the contribution $\Delta_{\nu_{\tau}}$ related to the heavy tau neutrino.

In the specific $S O(10)$ model we analyze in the present context, the two shifts are numerically given by

$$
\Delta_{\tau}=0.64 \times 10^{3} \mathrm{GeV}^{2} \text { and } \Delta_{\nu_{\tau}}=4.07 \times 10^{3} \mathrm{GeV}^{2} .
$$

These shifts are individually significantly larger than the typical errors of the slepton/sneutrino masses squared which are of order 0.1 to $0.2 \times 10^{3} \mathrm{GeV}^{2}$. The effects of both the tau and the neutrino tau Yukawa coupling can therefore be extracted experimentally.

We shall exploit these relations systematically to determine the GUT scale parameters and the right-handed neutrino mass in the framework of the error estimates for the charged and neutral sleptons of the first and third generation.

\section{(a) Electroweak SM D-terms}

The difference between $e$-sneutrino and L-selectron mass

$$
\begin{aligned}
m_{\tilde{\nu}_{e \mathrm{~L}}}^{2}-m_{\tilde{e}_{\mathrm{L}}}^{2} & =D_{\tilde{\nu}_{\mathrm{L}}}^{e l w}-D_{\tilde{e}_{\mathrm{L}}}^{e l w} \\
& =M_{\mathrm{W}}^{2} \cos 2 \beta
\end{aligned}
$$

measures the familiar D-terms associated with the $\mathrm{SU}(3) \times \mathrm{SU}(2) \times \mathrm{U}(1)$ symmetry breaking in the Standard Model. Radiative corrections are incorporated at the one-loop level in the numerical evaluation. The mass difference predicted by the electroweak D-terms and the radiative corrections,

$$
M_{\mathrm{W}}^{2} \cos 2 \beta+\text { rad.cor. }=-6.175 \times 10^{3} \mathrm{GeV}^{2},
$$

is well reproduced by the simulated mass measurements:

$$
m_{\tilde{\nu}_{e \mathrm{~L}}}^{2}-m_{\tilde{e}_{\mathrm{L}}}^{2}=-6.280_{-.260}^{+.255} \times 10^{3} \mathrm{GeV}^{2}
$$

This relation may serve as a consistency check for measurements. Here and in the next subsection the sneutrino mass error estimated in section 2 and the selectron mass errors

$$
m_{\tilde{e}_{\mathrm{R}}}=(125.32 \pm 0.05) \mathrm{GeV} \text { and } m_{\tilde{e}_{\mathrm{L}}}=190.0_{-0.3}^{+0.4} \mathrm{GeV}
$$

from Ref. [10] are used. 


\section{(b) Universal mass parameter $m_{16}$ and GUT D-term}

The most precise measurements of the soft mass term $m_{16}$ and the GUT D-term $D_{U}$ can be performed in the charged slepton sector:

$$
\begin{aligned}
3 m_{\tilde{e}_{\mathrm{R}}}^{2}+m_{\tilde{e}_{\mathrm{L}}}^{2} & =4 m_{16}^{2}+\left(3 \alpha_{R}+\alpha_{L}\right) M_{1 / 2}^{2}-\left(1 / 2+2 s_{\mathrm{W}}^{2}\right) M_{\mathrm{Z}}^{2} \cos 2 \beta \\
m_{\tilde{e}_{\mathrm{R}}}^{2}-m_{\tilde{e}_{\mathrm{L}}}^{2} & =4 D_{U}+\left(\alpha_{R}-\alpha_{L}\right) M_{1 / 2}^{2}+\left(1 / 2-2 s_{W}^{2}\right) M_{\mathrm{Z}}^{2} \cos 2 \beta
\end{aligned}
$$

The universal mass parameter $M_{1 / 2}$ is assumed to be pre-determined very precisely in the gaugino sector of the theory. In the numerical analysis the next-to-leading order is included properly. Using the estimated mass measurement errors, the fundamental parameters $m_{16}$ and $D_{U}$ at the high scale can be precisely determined:

$$
m_{16}=70.0_{-0.2}^{+0.3} \mathrm{GeV}, \quad D_{U}=0_{-40}^{+30} \mathrm{GeV}^{2} .
$$

The scalar mass parameter $m_{16}$ can be extracted with an accuracy significantly better than $1 \mathrm{GeV}$ while the square-root of the $\mathrm{SO}(10)$ D-term can be measured at the level of less than $10 \mathrm{GeV}$.

\section{(c) Yukawa interactions and seesaw scale}

As noticed in Refs. [23, 21], the heavy R-neutrino mass affects the evolution of the iso-doublet scalar mass parameters above the seesaw scale through the neutrino Yukawa couplings, but the iso-scalar parameters will be much less affected. Since the effect is induced by the Yukawa couplings, we anticipate that only the third generation will signal the seesaw scale.

To simplify the analysis, we eliminate the stau mixing parameters by adding up the 1 and 2 masses squared, or the $\mathrm{L}$ and $\mathrm{R}$ masses, correspondingly. Part of the difference between the stau and the selectron sector is induced by the $\tau$-Yukawa coupling as can easily be seen by inspecting the renormalization group equations (see e.g. [21] and references therein). This part can be projected out by using the sum rule

$$
2 \Delta_{\tau}=\left(m_{\tilde{e}_{\mathrm{L}}}^{2}+m_{\tilde{e}_{\mathrm{R}}}^{2}-m_{\tilde{\nu}_{\mathrm{L}} \mathrm{L}}^{2}\right)-\left(m_{\tilde{\tau}_{1}}^{2}+m_{\tilde{\tau}_{2}}^{2}-m_{\tilde{\nu}_{\tau \mathrm{L}}}^{2}\right)+2 m_{\tau}^{2} .
$$

Inserting the expected experimental errors for the masses from section 2 and Refs. [10, 22],

$$
\Delta_{\tau}=0.63_{-0.27}^{+0.28} \times 10^{3} \mathrm{GeV}^{2} .
$$

We conclude that the effect of the $\tau$ Yukawa coupling can be isolated at the 2- $\sigma$ level.

As we are primarily interested in identifying the effect of the right-handed neutrinos we consider the following sum rule for the $\Delta_{\nu_{\tau}}$ parameter derived from eqs. (33)-(38):

$$
2 \Delta_{\nu_{\tau}}\left[M_{\mathrm{R}_{3}}\right]=\left(3 m_{\tilde{\nu}_{\mathrm{eL}}}^{2}-m_{\tilde{e}_{\mathrm{L}}}^{2}-m_{\tilde{\mathrm{e}}_{\mathrm{R}}}^{2}\right)-\left(3 m_{\tilde{\nu}_{\tau \mathrm{L}}}^{2}-m_{\tilde{\tau}_{1}}^{2}-m_{\tilde{\tau}_{2}}^{2}\right)-2 m_{\tau}^{2} .
$$

This relation holds exactly at tree-level and gets modified by small corrections at the oneloop level. The particular form of eq. (50) implies that all D-terms (electroweak and GUTinduced) and the effects of the $\tau$ Yukawa coupling cancel. With the simulated mass measurement results one obtains

$$
\Delta_{\nu_{\tau}}=4.1_{-0.56}^{+0.55} \times 10^{3} \mathrm{GeV}^{2} .
$$




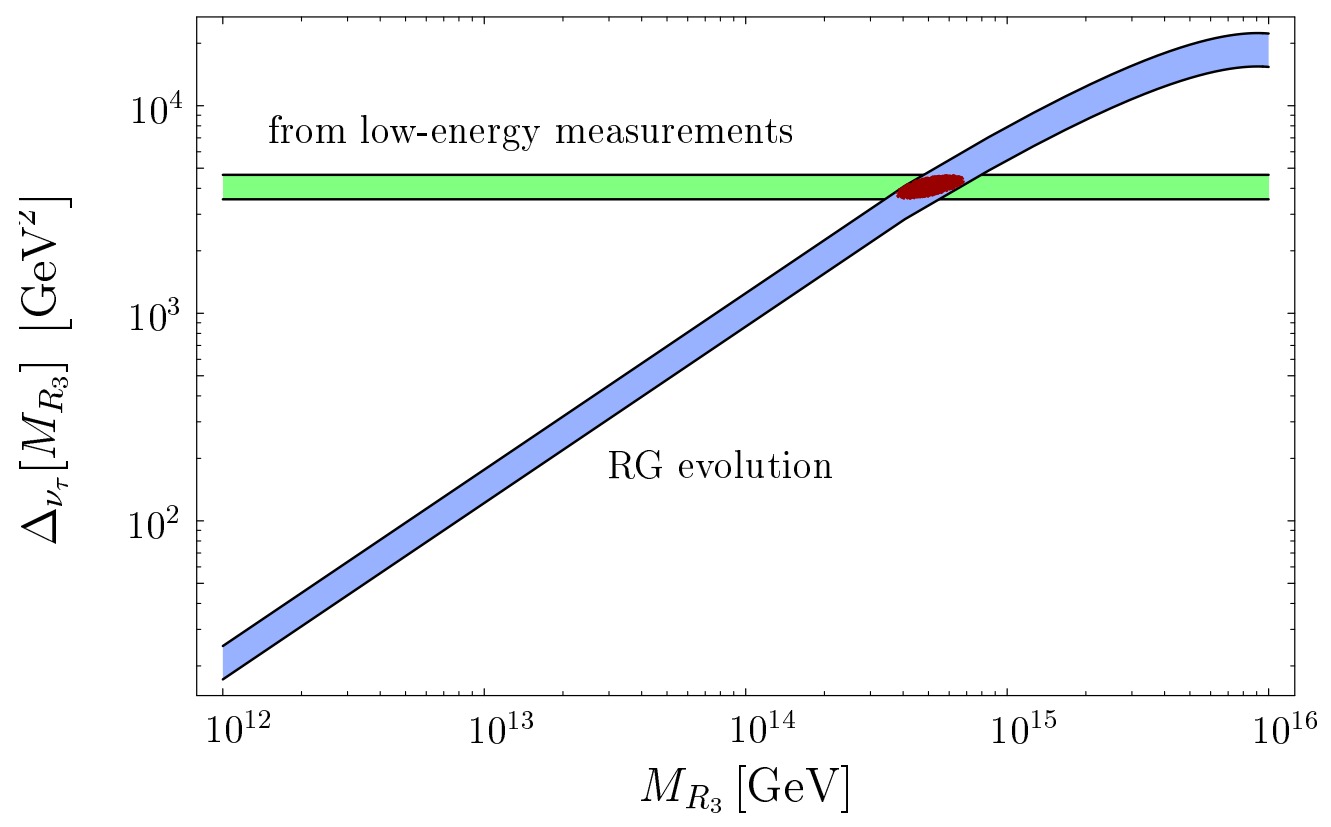

Figure 4: Shift $\Delta_{\nu_{\tau}}$ in the evolution of the tau-neutrino mass as calculated from the renormalization group equations, eq. (53) (blue band) and compared with low-energy mass measurements, eq. (51) (green band). The widths of the bands indicate estimated onestandard-deviations errors of the experimental input parameters. The red crossing region is the statistical combination which determines the neutrino seesaw scale $M_{\mathrm{R}_{3}}$ of the third generation.

It follows from the renormalization group equations that $\Delta_{\nu_{\tau}}\left[M_{\mathrm{R}_{3}}\right]$ is of the order $Y_{\nu}^{2} \log M_{G U T}^{2} / M_{\mathrm{R}_{3}}^{2}$. Since the Yukawa coupling $Y_{\nu}$ can be estimated in the seesaw mechanism by the mass $m_{\nu_{3}}$ of the third left-handed neutrino,

$$
Y_{\nu}^{2}=m_{\nu_{3}} M_{\mathrm{R}_{3}} /(v \sin \beta)^{2},
$$

the parameter $\Delta_{\nu_{\tau}}\left[M_{R_{3}}\right]$ depends approximately linearly on the mass $M_{\mathrm{R}_{3}}$,

$$
\Delta_{\nu_{\tau}}\left[M_{R_{3}}\right] \simeq \frac{m_{\nu_{3}} M_{\mathrm{R}_{3}}}{16 \pi^{2}(v \sin \beta)^{2}}\left(3 m_{16}^{2}+A_{0}^{2}\right) \log \frac{M_{G U T}^{2}}{M_{\mathrm{R}_{3}}^{2}},
$$

so that it can well be determined. [ $v$ and $\tan \beta$ are the usual parameters in the Higgs sector.] Inserting the pre-determined value for $m_{16}$ from the analyses above and the trilinear coupling $A_{0}$ from stop mixing [24], we can calculate $M_{\mathrm{R}_{3}}$, cf. Fig. 4. Assuming hierarchical neutrino masses, we have identified the error of the neutrino mass $m_{\nu_{3}}$ with the current uncertainty of the atmospheric neutrino mass difference [25]. With these assumptions, one obtains

$$
M_{\mathrm{R}_{3}}=3.7 \text { to } 6.9 \times 10^{14} \mathrm{GeV},
$$

to be compared with the initial value $M_{\mathrm{R}_{3}}=6 \times 10^{14} \mathrm{GeV}$. This analysis thus provides us with a unique estimate of the high-scale $\nu_{R}$ mass parameter $M_{\mathrm{R}_{3}}$. 


\section{Added Note}

As mentioned in footnote 4, we have up to now neglected the effect of lepton flavor violation (LFV) induced in the soft SUSY breaking parameters of the slepton sector due to the effect of neutrino Yukawa interactions in the RGE evolution of the parameters. These in turn lead to flavor violating lepton decays such as $\mu \rightarrow e \gamma[26]$. However, it has been shown in Ref. [20] that for the model under consideration the bounds due to rare lepton decays are not violated: $\operatorname{BR}(\mu \rightarrow e \gamma) \sim O\left(10^{-13}\right)$ and $\operatorname{BR}(\tau \rightarrow \mu \gamma) \sim O\left(10^{-8}\right)$. The latter is close to the latest experimental bound of $6.8 \times 10^{-8}[27]$ and it will be tested at the $B$ factories within the next few years. The former branching ratio will be scrutinized in a dedicated PSI experiment. A second class of observables can be determined in flavor violating decays of supersymmetric particles, see [28] and further references quoted in these papers. In particular, the off-diagonal terms in $M_{L}^{2}$ imply that every sneutrino can decay in principle into all charged leptons. However, when assuming that $Y_{\nu}\left(Y_{l}\right)$ has a structure similar to $Y_{u}$ $\left(Y_{d}\right)$ at $M_{G U T}$ as in the model under consideration, the flavor violation decays are suppressed

by powers of the CKM-matrix $V_{i j}$. This implies that our results are only weakly affected by the additional decay modes and the LFV decays reach at most $10 \%$. For the same reason the production of $\tilde{\nu}_{\mu}$ and $\tilde{\nu}_{\tau}$ in $e^{-} \gamma$ reactions is expected to be small. Clearly, the measurements of LFV sneutrino and slepton decays will provide additional information on the effects generated by $Y_{\nu}$ and, thus, can be used to scrutinize the physics at the see-scale $M_{\mathrm{R}_{3}}$ further.

\section{Summary}

In this report we have shown that chargino decays to sneutrinos and charged leptons, if kinematically allowed as two-body decays, provide an excellent opportunity to measure the masses of the L-sneutrinos in the three generations. Accuracies better than a per-cent can be expected when these masses are measured in chargino pair production at an $e^{+} e^{-}$ linear collider. [R-sneutrinos acquire masses near the GUT scale by the seesaw mechanism.] Independent cross-checks may be performed in the production of $e$-sneutrinos and charginos in high energy e $\gamma$ collisions.

Of particular interest is the comparison of scalar masses in the tau and the electron sector. If the scalar mass parameters are universal at the GUT scale, as in minimal supergravity, this regularity can be unraveled in the first and second generation of the scalar masses at the electroweak scale. However, universality will be broken between the first two and the third generation in theories incorporating the seesaw mechanism. The running of the masses from the GUT to the electroweak scale will be affected by loops involving the heavy Rneutrino with large Yukawa coupling in the third generation. Sum rules for mass differences of sneutrinos and selectrons between the first and third generation can be constructed that project out this contribution. Being approximately linear in the seesaw scale, the scale can be estimated from the sneutrino and slepton masses with good accuracy. In this way a method has been found by which the high R-neutrino mass can, at least indirectly, be measured. Thus, by means of extrapolations governed by the renormalization group, the high accuracy 
in the slepton and sneutrino mass measurements can be exploited to determine high-scale parameters that cannot be accessed directly.

\section{Acknowledgments}

We are grateful to H.-U. Martyn and D. E. Zerwas for numerous discussions on experimental aspects and background estimates. Moreover, special thanks go to H.-U. Martyn for his critical reading of the manuscript. W.P. is supported by a MCyT Ramon y Cajal contract, by the Spanish grant BFM2002-00345, by the European Commission Human Potential Program RTN network HPRN-CT-2000-00148 and partly by the Swiss 'Nationalfonds'. P.M.Z. thanks M.E. Peskin of SLAC and DFG for partial support; he gratefully acknowledges the warm hospitality extended to him during a stay at the Stanford Linear Accelerator Center SLAC where part of this study was worked out.

\section{References}

[1] K. S. Babu and R. N. Mohapatra, Phys. Rev. Lett. 70 (1993) 2845;

for a review see: S. F. King, Rept. Prog. Phys. 67 (2004) 107.

[2] P. Minkowski, Phys. Lett. B 67 (1977) 421;

M. Gell-Mann, P. Ramond, and R. Slansky, in Proc. of the Workshop on Complex Spinors and Unified Theories, Stony Brook, New York, North-Holland, 1979;

T. Yanagida, (KEK, Tsukuba), 1979;

R. N. Mohapatra and G. Senjanovic, Phys. Rev. Lett. 44 (1980) 912.

[3] A. Freitas, A. von Manteuffel and P. M. Zerwas, Eur. Phys. J. C 40, 435 (2005).

[4] U. Nauenberg, Contribution to the 3rd Workshop of the Extended ECFA/DESY Linear Collider Study, Prague, Czech Republic (2002).

[5] V. D. Barger, T. Han and J. Kelly, Phys. Lett. B 419 (1998) 233.

[6] B. C. Allanach et al., Eur. Phys. J. C 25 (2002) 113.

[7] Supersymmetry Parameter Analysis (SPA) Project, http://spa.desy.de/spa/.

[8] J. A. Aguilar-Saavedra et al. [ECFA/DESY LC Physics Working Group], arXiv:hepph/0106315;

T. Abe et al. [American Linear Collider Working Group], in Proc. of the APS/DPF/DPB Summer Study on the Future of Particle Physics (Snowmass 2001) ed. N. Graf, arXiv:hep-ex/0106056;

K. Abe et al. [ACFA Linear Collider Working Group], arXiv:hep-ph/0109166. 
[9] S. Y. Choi, A. Djouadi, M. Guchait, J. Kalinowski, H. S. Song and P. M. Zerwas, Eur. Phys. J. C 14 (2000) 535.

[10] A. Freitas, A. von Manteuffel and P. M. Zerwas, Eur. Phys. J. C 34 (2004) 487.

[11] J. H. Kühn, A. Reiter and P. M. Zerwas, Nucl. Phys. B 272 (1986) 560.

[12] S. Y. Choi, H.-U. Martyn and P. M. Zerwas, DESY 05-150 and hep-ph/0508021 [Eur. Phys. J. C in press].

[13] LHC/LC Study Group Working Report, eds. G. Weiglein et al., hep-ph/0410364; K. Desch, J. Kalinowski, G. Moortgat-Pick, M. M. Nojiri and G. Polesello, JHEP 0402, 035 (2004).

[14] H.-U. Martyn, LC Note LC-PHSM-2003-071, hep-ph/0406123.

[15] I. F. Ginzburg, G. L. Kotkin, V. G. Serbo and V. I. Telnov, JETP Lett. 34 (1981) 491 [Pisma Zh. Eksp. Teor. Fiz. 34 (1981) 514].

[16] V. A. Telnov, A Code for the Simulation of Luminosities and QED Backgrounds at Photon Colliders, presented at the ECFA-DESY Linear Collider Workshop, St. Malo, France, April 2002.

[17] A. F. Zarnecki, Acta Phys. Polon. B 34 (2003) 2741.

[18] C. F. Kolda and S. P. Martin, Phys. Rev. D 53 (1996) 3871.

[19] W. Buchmüller and D. Wyler, Phys. Lett. B 521 (2001) 291.

[20] A. Masiero, S. K. Vempati and O. Vives, Nucl. Phys. B 649 (2003) 189.

[21] G. A. Blair, W. Porod and P. M. Zerwas, Eur. Phys. J. C 27 (2003) 263.

[22] A. Freitas, H. U. Martyn, U. Nauenberg and P. M. Zerwas, in Proc. of the International Conference on Linear Colliders (LCWS 04), Paris, France, 19-24 Apr 2004 [hepph/0409129].

[23] H. Baer, C. Balazs, J. K. Mizukoshi and X. Tata, Phys. Rev. D 63 (2001) 055011.

[24] E. Boos, H. U. Martyn, G. Moortgat-Pick, M. Sachwitz, A. Sherstnev and P. M. Zerwas, Eur. Phys. J. C 30 (2003) 395;

A. Bartl, H. Eberl, S. Kraml, W. Majerotto, W. Porod and A. Sopczak, Z. Phys. C 76 (1997) 549 .

[25] M. Maltoni, T. Schwetz, M. A. Tortola and J. W. F. Valle, New J. Phys. 6 (2004) 122;

G. L. Fogli, E. Lisi, A. Marrone and A. Palazzo, hep-ph/0506083. 
[26] F. Borzumati and A. Masiero, Phys. Rev. Lett. 57 (1986) 961;

J. Hisano, T. Moroi, K. Tobe and M. Yamaguchi, Phys. Rev. D 53 (1996) 2442;

F. Deppisch, H. Päs, A. Redelbach, R. Rückl and Y. Shimizu, Eur. Phys. J. C 28 (2003) 365.

[27] B. Aubert et al. [BABAR Collaboration], hep-ex/0502032.

[28] N. Arkani-Hamed, H. C. Cheng, J. L. Feng and L. J. Hall, Phys. Rev. Lett. 77, 1937 (1996) and Nucl. Phys. B 505, 3 (1997);

J. Hisano, M. M. Nojiri, Y. Shimizu and M. Tanaka, Phys. Rev. D 60, 055008 (1999); W. Porod and W. Majerotto, Phys. Rev. D 66 (2002) 015003;

F. Deppisch, H. Päs, A. Redelbach, R. Rückl and Y. Shimizu, Phys. Rev. D 69 (2004) 054014;

F. Deppisch, H.-U. Martyn, H. Päs, A. Redelbach and R. Rückl, in Proc. of the International Conference on Linear Colliders (LCWS 04), Paris, France, 19-24 Apr 2004 [hep-ph/0408140];

W. Porod, hep-ph/0410318. 